\title{
Calculation of Fully Anisotropic Liquid Crystal Waveguide Modes
}

\author{
Jeroen Beeckman, Richard James, F. Aníbal Fernández, Member, IEEE, Wout De Cort, Pieter J. M. Vanbrabant, and \\ Kristiaan Neyts
}

\begin{abstract}
The accurate analysis of optical waveguides is an important issue when designing devices for optical communication. Waveguides combined with liquid crystals have great potential because they allow waveguide tuning over a wide range using low voltages. In this paper, we present calculations that combine an advanced algorithm for calculating liquid crystal behavior and a finite-element mode solver that is able to incorporate the full anisotropy of the materials. Calculation examples demonstrate the validity of our program.
\end{abstract}

Index Terms-Finite-element mode solver, nematic liquid crystal, optical waveguides.

\section{INTRODUCTION}

W AVEGUIDES are used to guide electromagnetic signals from one point to another and they form the backbone of communication systems. Optical waveguides can be made in silica, such as in optical fibers, or in other material systems such as Silicon-on-Insulator, InP, InGaAs, etc. Light is captured in the high index material of the core. The vast majority of these materials do not allow simple tuning of the waveguide properties. Often, temperature tuning is considered, because voltage tuning is not possible. The reason lies in the fact that the electro-optic coefficients of most crystals are very small. Liquid crystals on the other hand have excellent electro-optic properties, and due

Manuscript received November 28, 2008; revised February 10, 2009. First published May 02, 2009; current version published August 14, 2009. The work of W. De Cort was supported by the Institute for the Promotion of Innovation through Science and Technology in Flanders (IWT-Vlaanderen). The work of J. Beeckman was supported by a mobility grant from the Research Foundation Flanders (FWO-Vlaanderen) for conducting the research at University College London. This work has been carried out in the framework of the IAP project Photonics@be of the Belgian Science Policy.

J. Beeckman and P. J. M. Vanbrabant are with the Liquid Crystals and Photonics Group, Department of Electronics and Information Systems, Ghent University, 9000 Gent, Belgium, and also with the Research Foundation Flanders (FWO), B-1000 Brussels, Belgium (e-mail: jeroen.beeckman@elis.ugent.be; pieter.vanbrabant@elis.ugent.be).

R. James and F. A. Fernández are with the Department of Electronics and Electrical Engineering, University College London, London WC1E 7JE, U.K. (e-mail: r.james@ee.ucl.ac.uk; a.fernandez@ee.ucl.ac.uk).

W. De Cort is with the Liquid Crystals and Photonics Group, Department of Electronics and Information Systems, Ghent University, 9000 Gent, Belgium, and also with the Photonics Research Group, Department of Information Technology, Ghent University, 9000 Gent, Belgium (e-mail: wout.decort@ ugent.be).

K. Neyts is with the Liquid Crystals and Photonics Group, Department of Electronics and Information Systems, Ghent University, 9000 Gent, Belgium (e-mail: kristiaan.neyts@elis.ugent.be).

Color versions of one or more of the figures in this paper are available online at http://ieeexplore.ieee.org

Digital Object Identifier 10.1109/JLT.2009.2016673 to their large electro-optic coefficient they are nowadays massively used in display applications. For this reason, many groups have proposed configurations in which moderate voltages are used to change the orientation of the liquid crystal, which in turn alters the properties of the waveguide. The liquid crystal can be used either as a tunable cladding material [1]-[5] or as the guiding material itself [6], [7]. Even the large nonlinearity of the liquid crystal can be exploited to form self-induced waveguides [8]-[10]. Moreover, commercial devices are currently used in long haul fiber communication networks.

Nematic liquid crystals are organic, anisotropic liquids that are characterized by a degree of orientational, but not positional order while still preserving the mobility of liquids. The molecules are typically elongated and the orientational order leads to uniaxial properties. Due to the anisotropy of the dielectric properties, the molecules tend to align either along or perpendicular to externally applied electric fields. This means that the orientation of the molecules can be changed by voltages applied at electrodes. This change in orientation induces a change in the optical properties. The modeling of the liquid crystal orientation in a complex geometry is a difficult problem because a number of nonlinear equations need to be solved simultaneously. In this paper, a variable order calculation [11] was used based on the minimization of the Landau-de Gennes free energy functional [12]. The model is implemented in a finite-element scheme. Due to the incorporation of the variable degree of order of the liquid crystal, the program is able to model disclinations where there is a rapid variation in the order parameter. When liquid crystals are used in combination with for example rib waveguides, the behavior of the liquid crystal near edges can be modeled in an accurate way with our simulation tool.

Optical modeling of the liquid crystal is complicated by the optical anisotropy and non-uniformity. The optical tensor $\bar{\varepsilon}$ in the general case can contain up to six nonzero different components. Often the problem is handled with some approximations in terms of the anisotropy or in terms of dimensionality [7], [13], [14]. Transverse anisotropy means that $\varepsilon_{x z}$ and $\varepsilon_{y z}$ are zero, while the incorporation of longitudinal anisotropy means that also these terms can be nonzero. Longitudinal anisotropy leads to off-axis energy transfer of the extraordinary polarization component [15]-[17]. The ordinary polarization component does not exhibit an off-axis deviation. A number of modesolver algorithms have been proposed in the past in which only transverse anisotropy has been included [18]-[25]. Other algorithms do include full anisotropy of the dielectric tensor and a classification can be made in terms of the fields that are used to 
formulate the eigenvalue equation. In [26], [27] the eigenvalue problem is formulated in terms of all electric and magnetic field components $\left\{E_{t}, H_{t}, E_{z}, H_{z}\right\}$, while in [28] only the transverse components of both fields are used $\left\{E_{t}, H_{t}\right\}$. In [29] and [30], the magnetic field components are used $\left\{H_{t}, H_{z}\right\}$. A formulation in terms of the electric fields $\left\{E_{t}, E_{z}\right\}$ is presented in [31], but no results are presented for a full anisotropy of $\bar{\varepsilon}$. Recently a formulation in terms of the electric fields including higher order edge elements is presented for the analysis of photonic crystals with full anisotropy, but the wave number $\bar{k}$ is treated as an input parameter while the frequency is calculated [32]. In this paper, we have implemented a full vectorial mode solver in terms of the electric fields with the frequency as an input. Higher order edge elements are used, which offer a higher computation efficiency compared to previous works and avoids spurious modes. The strength of our simulation is that it is directly coupled with an advanced liquid crystal calculation [11].

In Section II, the theory and algorithms that are used for the simulation program are discussed. Section III assesses the accuracy of the numerical model, through comparison with theoretical results for an anisotropic slab waveguide, while Section IV shows results for a rib waveguide. In Section $\mathrm{V}$ the modes of a rib waveguide surrounded by a liquid crystal cladding are calculated and analyzed.

\section{Finite-Element Mode Solver}

The mode solver is based on the solution of the Helmholtz equation for the electric field

$$
\nabla \times\left(\frac{1}{\mu_{r}} \nabla \times \bar{E}\right)-k_{0}^{2} \overline{\bar{\varepsilon}} \cdot \bar{E}=0 \quad \text { in } \Omega
$$

and the boundary condition

$$
\bar{n} \times \bar{E}=0 \quad \text { on } \partial \Omega
$$

equivalent to a perfect electric conductor. The corresponding variational problem is given by [33]

$$
\left\{\begin{array}{l}
\delta F(\bar{E})=0 \\
\bar{n} \times \bar{E}=0 \quad \text { on } \partial \Omega
\end{array}\right.
$$

where

$$
F(\bar{E})=\frac{1}{2} \iint_{\Omega}\left[\frac{1}{\mu_{r}}(\nabla \times \bar{E}) \cdot(\nabla \times \bar{E})^{*}-k_{0}^{2} \bar{E} \cdot \overline{\bar{\varepsilon}} \cdot \bar{E}^{*}\right] d \Omega .
$$

Since modes that propagate along the $z$ direction are sought, the field is assumed to be of the form $\bar{E}(x, y, z)=$ $\bar{E}(x, y) \exp \left(-j k_{z} z\right)$, which yields

$$
\begin{aligned}
F(\bar{E})= & \frac{1}{2} \iint_{\Omega}\left[\frac{1}{\mu_{r}}\left(\nabla_{t} \times \bar{E}\right) \cdot\left(\nabla_{t} \times \bar{E}\right)^{*}-k_{0}^{2} \bar{E} \cdot \overline{\bar{\varepsilon}} \cdot \bar{E}^{*}\right. \\
& \left.+\frac{1}{\mu_{r}}\left(\nabla_{t} E_{z}+j k_{z} \bar{E}_{t}\right) \cdot\left(\nabla_{t} E_{z}+j k_{z} \bar{E}_{t}\right)^{*}\right] d \Omega .
\end{aligned}
$$

In this equation, $\nabla_{t}$ denotes the transverse nabla operator containing only $x$ and $y$ derivatives. $\bar{E}_{t}$ and $E_{z}$ denote the transverse and longitudinal component of the field, respectively. Applying the substitutions $\bar{e}_{t}=k_{z} \bar{E}_{t}$ and $e_{z}=-j E_{z}$ and multiplying by $k_{z}^{2}$ gives

$$
\begin{aligned}
F=\frac{1}{2} \iint_{\Omega}[ & \frac{1}{\mu_{r}}\left(\nabla_{t} \times \bar{e}_{t}\right) \cdot\left(\nabla_{t} \times \bar{e}_{t}\right)^{*}-k_{0}^{2} \bar{e}_{t} \cdot \overline{\bar{\varepsilon}}_{t t} \cdot \bar{e}_{t}^{*} \\
& +\frac{k_{z}^{2}}{\mu_{r}}\left(\nabla_{t} e_{z} \cdot \nabla_{t} e_{z}^{*}+\bar{e}_{t} \cdot \nabla_{t} e_{z}^{*}+\nabla_{t} e_{z} \cdot \bar{e}_{t}^{*}+\bar{e}_{t} \cdot \bar{e}_{t}^{*}\right) \\
& +j k_{0}^{2} k_{z}\left(\bar{e}_{t} \cdot \bar{\varepsilon}_{t z} e_{z}^{*}-e_{z} \bar{\varepsilon}_{z t} \cdot \bar{e}_{t}^{*}\right) \\
& \left.-k_{0}^{2} k_{z}^{2} e_{z} \varepsilon_{z z} e_{z}\right] d \Omega .
\end{aligned}
$$

The domain is meshed by triangular elements, and the field components discretized by $\bar{e}_{t}^{e}=\sum_{i} \bar{N}_{i}^{e} e_{t i}^{e}$ and $e_{z}^{e}=\sum_{i} L_{i}^{e} e_{z i}^{e}$, where $\bar{N}_{i}^{e}$ and $L_{i}^{e}$ are the local shape functions [34]. Finally the Rayleigh-Ritz procedure [33] is applied to obtain a matrix eigenvalue system that can be solved for the propagation constant $k_{z}$.

$$
\begin{aligned}
{\left[\begin{array}{cc}
A_{t t} & 0 \\
0 & 0
\end{array}\right]\left[\begin{array}{l}
e_{t} \\
e_{z}
\end{array}\right]=-k_{z}^{2}\left[\begin{array}{ll}
B_{t t} & B_{t z} \\
B_{z t} & B_{z z}
\end{array}\right]\left[\begin{array}{l}
e_{t} \\
e_{z}
\end{array}\right] } \\
-k_{z}\left[\begin{array}{cc}
0 & j C_{t z} \\
-j C_{z t} & 0
\end{array}\right]\left[\begin{array}{l}
e_{t} \\
e_{z}
\end{array}\right]
\end{aligned}
$$

in which

$$
\begin{aligned}
& {\left[A_{t t}^{e}\right]=\iint\left[\frac{1}{\mu_{r}}\left\{\nabla_{t} \times \bar{N}^{e}\right\} \cdot\left\{\nabla_{t} \times \bar{N}^{e}\right\}^{T}\right.} \\
&\left.-k_{0}^{2}\left\{\bar{N}^{e}\right\} \cdot \overline{\bar{\varepsilon}}_{t t \cdot} \cdot\left\{\bar{N}^{e}\right\}^{T}\right] d \Omega \\
& {\left[B_{t t}^{e}\right]=\iint\left[\frac{1}{\mu_{r}}\left\{\bar{N}^{e}\right\} \cdot\left\{\bar{N}^{e}\right\}^{T}\right] d \Omega } \\
& {\left[B_{t z}^{e}\right]=} {\left[B_{z t}^{e}\right]^{T}=\iint\left[\frac{1}{\mu_{r}}\left\{\bar{N}^{e}\right\} \cdot\left\{\nabla_{t} L^{e}\right\}^{T}\right] d \Omega } \\
& {\left[B_{z z}^{e}\right]=\iint\left[\frac{1}{\mu_{r}}\left\{\nabla_{t} L^{e}\right\} \cdot\left\{\nabla_{t} L^{e}\right\}^{T}\right.}\left.\quad-k_{0}^{2} \varepsilon_{z z}\left\{L^{e}\right\}\left\{L^{e}\right\}^{T}\right] d \Omega \\
& {\left[C_{t z}^{e}\right]=} {\left[C_{z t}^{e}\right]^{T}=k_{0}^{2} \iint\left[\left\{\bar{N}^{e}\right\} \cdot \bar{\varepsilon}_{t z}\left\{L^{e}\right\}^{T}\right] d \Omega . }
\end{aligned}
$$

This equation is a quadratic eigenvalue problem [35], which simply reduces to a linear eigenvalue problem in the case of pure transverse anisotropy. In the general case, this quadratic eigenvalue problem can be reduced to a linear system by doubling the number of unknowns [35]. The array of unknowns $\left\{e_{t}^{e} e_{z}^{e}\right\}$ is extended by $\left\{k_{z} e_{t}^{e} k_{z} e_{z}^{e}\right\}$. The matrices may be extended in a number of ways, but in order to maintain the symmetry of the right hand side matrix the following form is used

$$
\begin{aligned}
& {\left[\begin{array}{cccc}
0 & 0 & -B_{t t} & -B_{t z} \\
0 & 0 & -B_{t z}^{T} & -B_{z z} \\
A_{t t} & 0 & 0 & -j C_{t z} \\
0 & 0 & j C_{t z}^{T} & 0
\end{array}\right]\left[\begin{array}{c}
e_{t} \\
e_{z} \\
k_{z} e_{t} \\
k_{z} e_{z}
\end{array}\right]} \\
& \quad=k_{z}\left[\begin{array}{cccc}
-B_{t t} & -B_{t z} & 0 & 0 \\
-B_{t z}^{T} & -B_{z z} & 0 & 0 \\
0 & 0 & -B_{t t} & -B_{t z} \\
0 & 0 & -B_{t z}^{T} & -B_{z z}
\end{array}\right]\left[\begin{array}{c}
e_{t} \\
e_{z} \\
k_{z} e_{t} \\
k_{z} e_{z}
\end{array}\right] .
\end{aligned}
$$


Alternatively, the longitudinal $\left(e_{z}\right)$ components from (7) may be eliminated, resulting in the following quadratic eigenvalue problem:

$$
\begin{array}{r}
\left(A_{t t}-C_{t z} B_{z z}^{-1} C_{z t}\right) e_{t}=k_{z}^{2}\left(-B_{t t}+B_{t z} B_{z z}^{-1} B_{z t}\right) e_{t} \\
+j k_{z}\left(B_{t z} B_{z z}^{-1} C_{z t}-C_{t z} B_{z z}^{-1} B_{z t}\right) e_{t}
\end{array}
$$

Similarly, via extension, this form may be reduced to a linear eigenvalue problem. Although this second method produces matrices of smaller order, the first method is preferable because the sparsity of the matrices involved is maintained, allowing for a shorter calculation time.

For the discretization, the cross-section $\Omega$ is subdivided into triangular elements using hybrid edge/nodal elements. Edge elements are used for the transverse fields, while the longitudinal field component is represented by nodal elements. Three types of finite-element discretizations have been implemented: constant tangential and linear normal (CT/LN), linear tangential and linear normal (LT/LN) and linear tangential and quadratic normal (LT/QN) vector basis functions [34]. The use of these vector elements greatly reduces the appearance of spurious modes in the eigenvalue calculation [36].

The disadvantage of the transformation for full anisotropy is a doubling in length of the solution vector. Associated with this is an increase in the number of degrees of freedom and memory requirements. This doubling can be avoided by using an iterative scheme to solve the quadratic eigenvalue problem [30] or by rearranging the equation to give an eigenvalue problem with an eigenvalue of $k_{0}^{2}$. The expanded eigensystem is free of spurious solutions and the matrices involved are highly sparse. There are a number of non-valid solutions with eigenvalues zero for which $E_{t}=0$. These non-valid solutions must not be confused with any kind of spurious modes since their origin is an algebraic transformation [31]. These are trivial to recognize and discount. In the expanded system the number of non-valid solutions is twice the number of unknowns in $E_{z}$. Compared with the reduced system that arises in the case of purely transverse anisotropy, the expanded system gives twice the number of modes, corresponding to both forward and backwards propagating modes. Due to the reciprocity of the liquid crystal the field patterns for these modes is identical.

Representation in terms of the magnetic field components is often preferred in finite-element calculations using nodal elements. This is because for optical waveguiding situations all magnetic field components are continuous across material interfaces (assuming all materials involved having the same magnetic permeability). Electric field components normal to the interfaces will be discontinuous and in nodal finite element calculations this will require a special treatment of these interfaces. Nodes must be doubled at interfaces to allow for the aforementioned discontinuity. A vector finite-element representation can be made to satisfy continuity of the field components tangential to element sides, so if these are chosen on interfaces, they will satisfy the correct boundary condition for $\mathrm{E}$ and $\mathrm{H}$ fields. No implication is made on the normal components and this allows the discontinuity of the normal electric field. Under these circumstances, electric fields are the most natural choice.

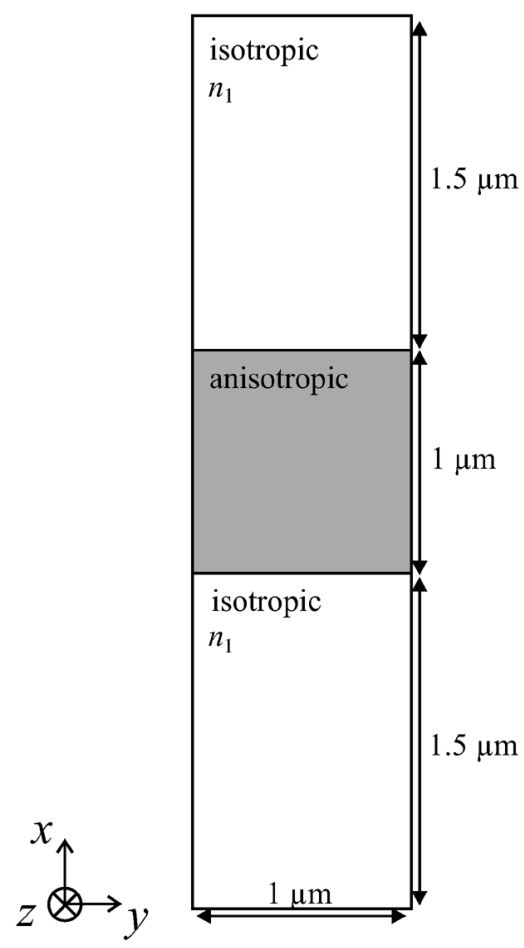

Fig. 1. Geometry of the slab waveguide.

\section{ONE-DimENSIONAL STRUCTURE}

In order to assess the accuracy of the numerical model, modes of an anisotropic slab waveguide have been calculated both theoretically and using the finite-element mode solver. The structure, a symmetric slab waveguide, is shown in Fig. 1. The optical parameters are uniform in each region and periodic boundary conditions have been implemented so that the structure is one dimensional. Since we are interested in a case where longitudinal anisotropy appears, in the Appendix the configuration with nonzero $\varepsilon_{x z}$ and zero $\varepsilon_{x y}$ and $\varepsilon_{y z}$ is considered. In this case, the dispersion relation (18) can be extracted analytically. Only the TM calculation is carried out because the TE modes in this case are the same as in the isotropic case with refractive index $\sqrt{\varepsilon_{y y}}$. Calculations were carried out for $\lambda=1 \mu \mathrm{m}$ and $n_{1}=1.5$.

For the sake of simplicity a uniaxial core material with $n_{\perp}=$ 1.55 and $n_{\|}=1.8$ is considered in this example. Several calculations were performed, varying the angle $\theta$ of the optic axis with respect to the $z$ axis in the range from $0^{\circ}$ to $90^{\circ}$. For $45^{\circ}$ the waveguide is monomode and the effective index of the TM0 mode is 1.638595 , calculated with the analytic formula (18). The comparison of the results obtained with the finite element solver [see Fig. 2(a)], shows that the value obtained with the CTLN elements converges to the same value as the LTQN elements. However, with the LTQN elements, the effective index converges faster to the desired solution as the number of degrees of freedom are increased (the number of degrees of freedom is equal to the sum total of nodal and edge variables). The superiority of the LTQN elements is in agreement with previous results [34] in isotropic media.

In Fig. 2(b) the values of the effective index as a function of the angle $\theta$ are plotted for LTQN elements with 1576 degrees of freedom. The theoretical values, obtained with (18) are also 
(a)

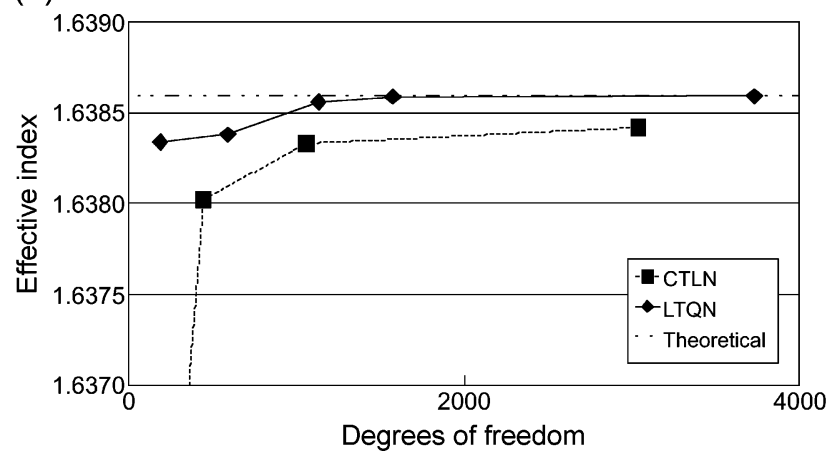

(b)

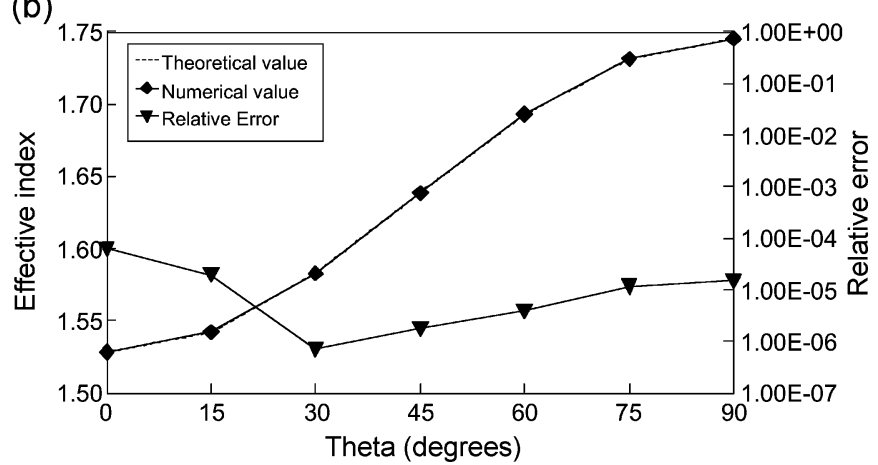

Fig. 2. Effective index of the modes of an anisotropic slab waveguide for the TM0 mode. (a) Effective index obtained for $45^{\circ}$ orientation of the long axis for different degrees of freedom. (b) Effective index in function of the angle of the long axis, calculated for LTQN elements (1576 degrees of freedom) and calculated with the analytic formula (18).

plotted and the two curves are indistinguishable. The relative error compared to the theoretical value remains below $6 \times 10^{-5}$. The modes obtained with the finite element solver show a linear increase in phase along the $x$ direction in the core layer as is expected theoretically from (17).

\section{Simple ANisotropic Rib WAVEguide}

The second example is a simple square waveguide. The core guiding layer consists of a uniaxial material with $n_{o}=1.55$, $n_{e}=1.8$ with the optic axis making an angle of $45^{\circ}$ with respect to the $z$ axis. The dimensions are shown in Fig. 3(a). In Fig. 3(b) the effective index of the fundamental mode is shown as a function of the degrees of freedom. It is clear that also for anisotropic cases the higher order LTQN elements are superior. Even with few elements, the effective index is very close to the final value.

\section{Rib Waveguide With Liquid CRystal Cladding}

In order to show the full capabilities of the numerical model, we consider a rib waveguide of 1 by $1 \mu \mathrm{m}$ surrounded by liquid crystal material as in Fig. 4. Two uniform electrodes apply a voltage across the liquid crystal layer. At zero voltage the liquid crystal is oriented along the $z$ axis. There is a pretilt of $2^{\circ}$ at the upper surface of the liquid crystal layer and there is no pretilt at the lower boundary surface. From a technological viewpoint, this can be achieved by using conventional rubbing of the upper surface and polarized UV curing of the alignment layer for the
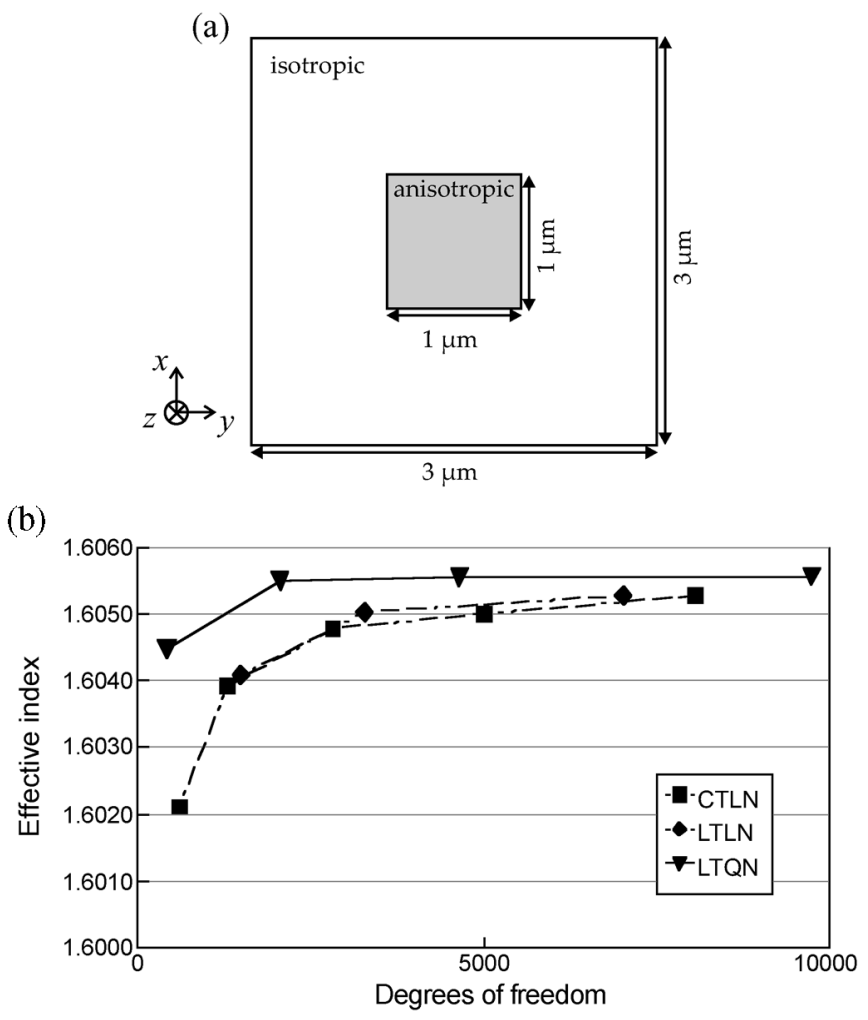

Fig. 3. (a) Geometry of the simple rib waveguide structure (b) and effective index of the fundamental mode for different degrees of freedom and different shape functions.

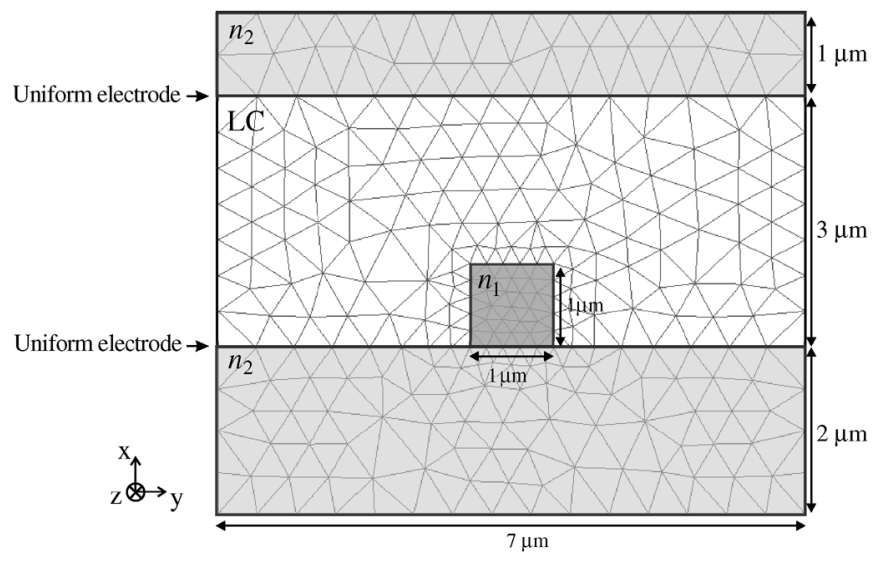

Fig. 4. Geometry of the rib waveguide with LC cladding.

lower surface, [37]. Additionally the corners of the rib waveguide also tend to induce longitudinal alignment [38]. Simulations have been performed for parameters of the liquid crystal ZLI-4972 [39] $\left(n_{o}=1.479, n_{e}=1.573, \varepsilon_{\perp}=3.1, \varepsilon_{\|}=8.3\right.$, $K_{11}=13.2 \mathrm{pN}, K_{22}=6.5 \mathrm{pN}$, and $K_{33}=18.3 \mathrm{pN}$ ). The refractive index of the core $n_{1}=1.7$ and the cladding $n_{2}=1.5$. These values of refractive index are typical of materials used in polymer waveguides.

The results of the liquid crystal calculation are shown in Fig. 5. For $0 \mathrm{~V}$, the liquid crystal layer has a tilt angle decreasing from the pretilt value to zero from top to bottom. In this case the quasi-TM and quasi-TE modes are nearly degenerate because the configuration is nearly the same along 

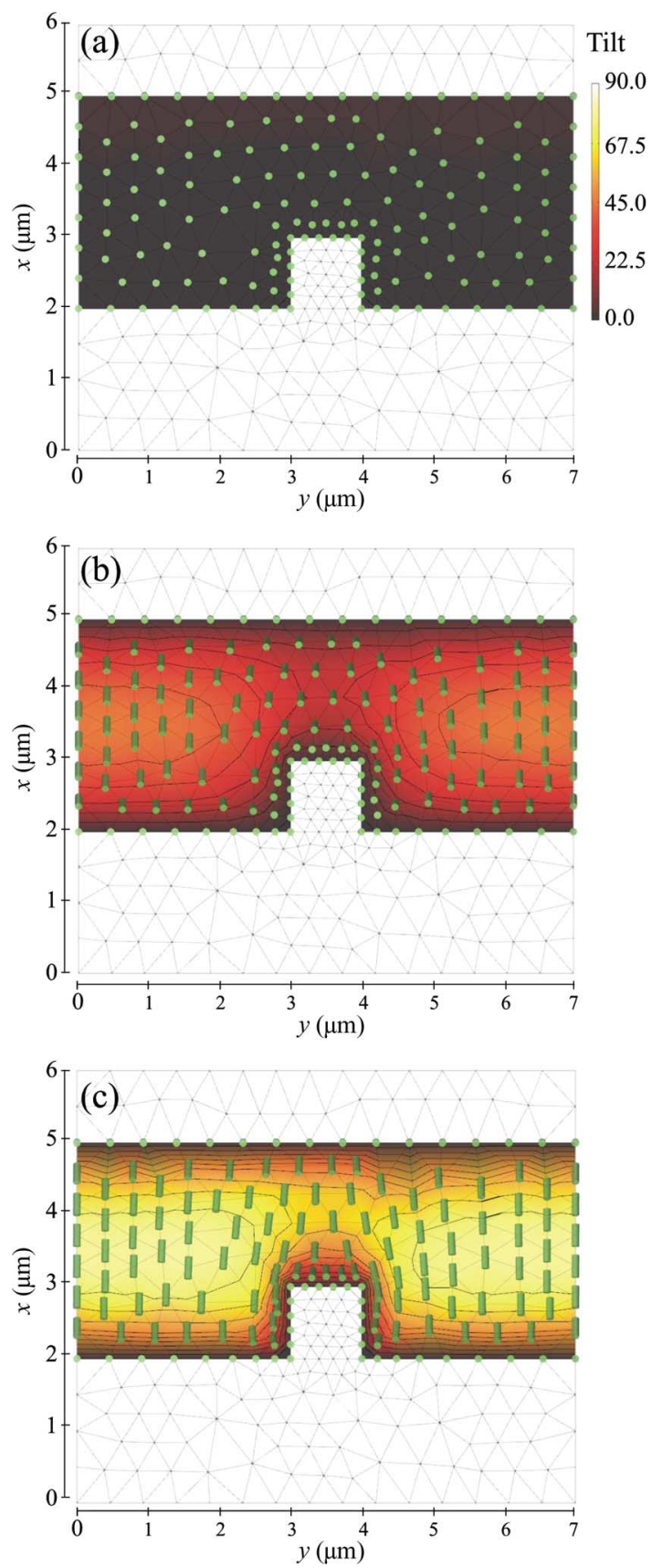

Fig. 5. Orientation of the liquid crystal in a rib waveguide for (a) $0 \mathrm{~V}$, (b) $2 \mathrm{~V}$, and (c) $4 \mathrm{~V}$. The background color indicates the tilt angle of the director as denoted by the colorbar.

the $x$ and $y$ directions, mainly due to the fact that the governing refractive index of the liquid crystal layer is $n_{o}$, which is close to the refractive index of the bottom layer. When the voltage is increased from 0 to $2 \mathrm{~V}$, the liquid crystal reorients reaching a maximum tilt of approximately $45^{\circ}$ in the middle of the layer. Above the rib waveguide the maximum tilt is smaller (about $30^{\circ}$ ), due to the voltage drop across the dielectric region of the rib waveguide. Reorientation of the liquid crystal has the strongest influence on the effective index in the vicinity of the waveguide core. However, in this region reorientation is restricted by anchoring forces. For $4 \mathrm{~V}$ the tilt reaches a maximum of $80^{\circ}$ throughout the layer and $65^{\circ}$ above the rib waveguide. Due to the anisotropy, a difference will be induced between the $x$ and $y$ polarized mode. The $x$ polarized mode will see a larger index of refraction while the $y$ polarized mode will not see this increase.

Fig. 6 shows the mode profiles for different voltages. For zero voltage, the TM mode profile is symmetric, because the index seen by the transverse fields is $n_{o}$, which is close to the refractive index of the bottom layer. For $10 \mathrm{~V}$ the TM mode extends more into the liquid crystal layer because the $x$ polarized field sees a refractive index $n_{e}$.

Fig. 7 shows the evolution of the effective index of the TM and the TE mode as a function of the applied voltage. One can see clearly that the effective index of the TE mode remains approximately the same while the effective index of the TM mode increases with increasing voltage. This is to be expected because the $x$ polarization sees $n_{o}$ for low voltages and $n_{e}$ for high voltages. For voltages around $6 \mathrm{~V}$ the director orientation in the bulk is nearly parallel to the $x$ axis. However the effective index still increases for higher voltages. This is due to the increase in tilt of the liquid crystal in the neighborhood of the rib waveguide. The gradient in the tilt of the molecules near the edges of the rib waveguide becomes steeper with increasing voltage. The change in effective index that we achieve is 0.015 for a liquid crystal with a birefringence of 0.094 , this means roughly $15 \%$ of the liquid crystal birefringence. The ratio of the change in effective index over the liquid crystal birefringence is strongly related to the relative amount of power of the mode that is present in the liquid crystal cladding.

Note that the TE mode shows a small decrease in effective index. This is because the $z$ component of the field sees $n_{e}$ for low voltages and $n_{o}$ for high voltages. When the refractive index contrast between the waveguide and cladding increases, the amplitude of the $z$ component of the field increases relative to the $x$ component. As a result, the influence of the $z$ component of the optical tensor becomes more important in comparison to the evolution of the $y$ component.

\section{CONCLUSION}

A computational model that predicts both the liquid crystal orientation and the optical mode properties of waveguides containing liquid crystals has been presented. Comparison with analytic results proves the validity of our model. Results for a rib waveguide surrounded by liquid crystal material show that the effective index of the TM mode can be tuned over a wide range, which is limited mainly by the birefringence of the liquid crystal. The program is freely available at http://www. elis.ugent.be/ELISgroups/lcd/research/modesolver.php.

\section{APPENDIX}

\section{Modes of AN ANISOTROPIC SLAB WAVEGUIDE}

For a slab waveguide, there is no variation in the $y$ direction. Assuming that the wave propagates in the $z$ direction, we insert $\bar{E}(x, z)=\bar{e}(x) \exp \left(-j k_{z} z\right)$ and $\bar{H}(x, z)=\bar{h}(x) \exp \left(-j k_{z} z\right)$ into Maxwell's equations. In the case of full anisotropy (a full tensor $\overline{\bar{\varepsilon}}$ ) it is not possible to split the problem into a pure TM $\left(e_{x}, h_{y}, e_{z}\right)$ and a TE $\left(h_{x}, e_{y}, h_{z}\right)$ part and the modes will be hybrid in nature [40]. Separation is only possible when the elements $\varepsilon_{x y}$ and $\varepsilon_{y z}$ are zero. This allows for longitudinal 

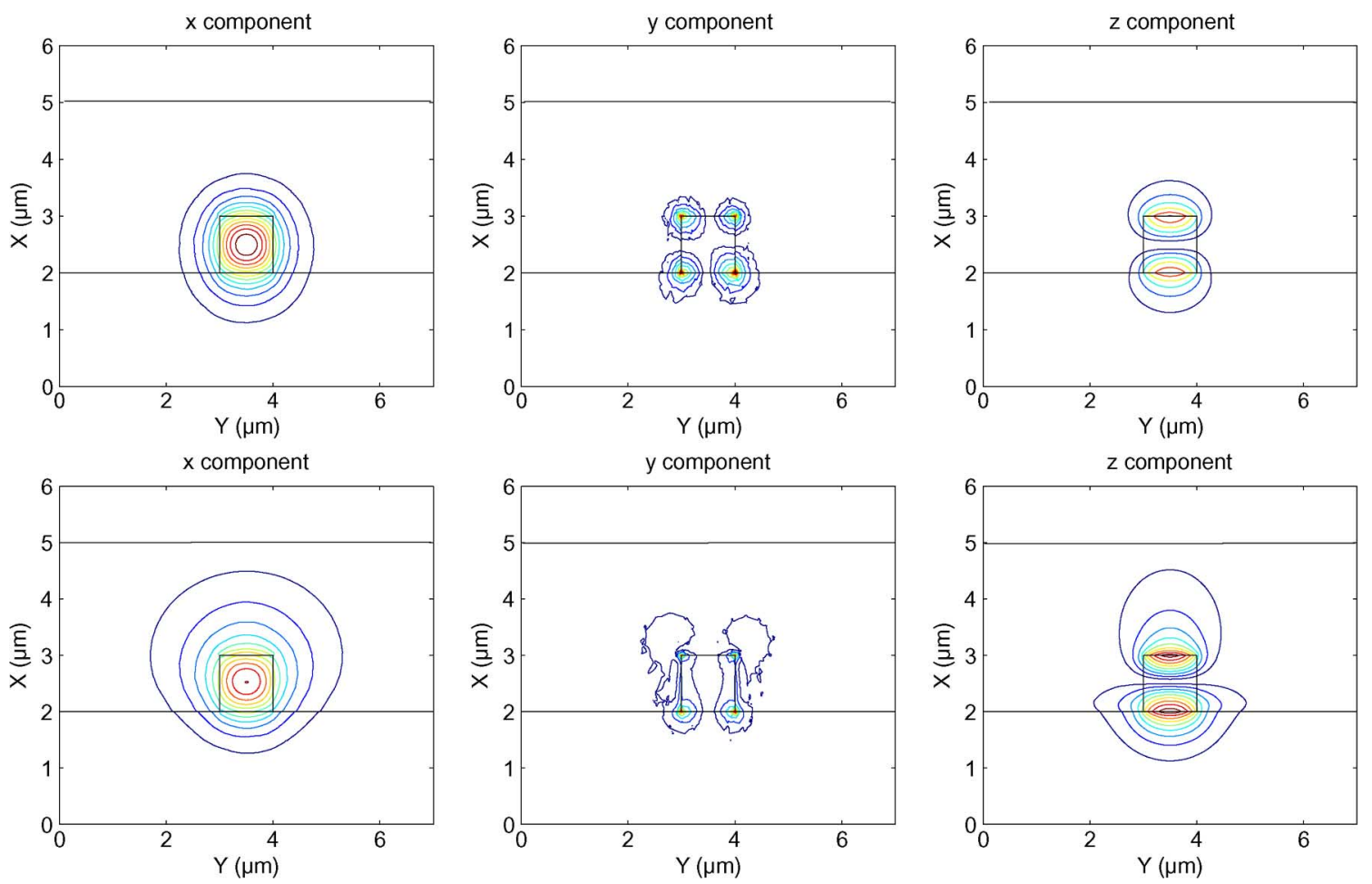

Fig. 6. TM mode profiles for an applied voltage of $0 \mathrm{~V}$ (top) and $10 \mathrm{~V}$ (bottom).

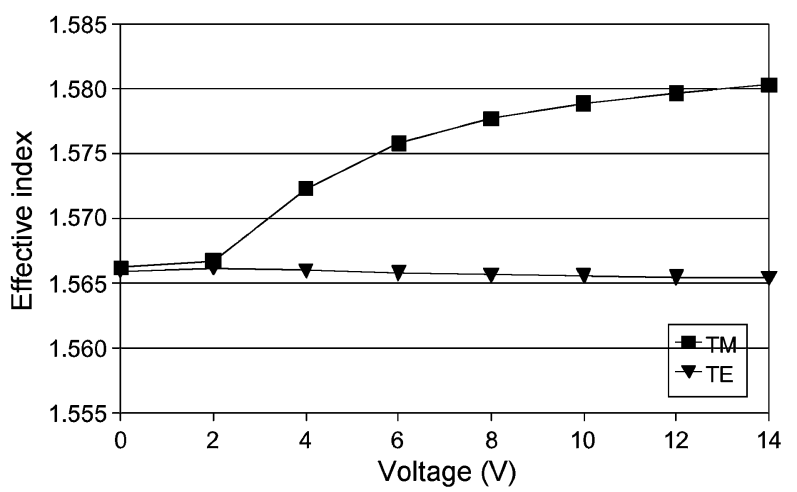

Fig. 7. Evolution of the effective index of the TM and the TE mode in function of applied voltage.

anisotropy, which we use for comparison with the numerical results. In this case, the TE problem is the same as in the isotropic case, with the refractive index determined by $\varepsilon_{y y}$. For the TM problem, the governing equations are

$$
\begin{aligned}
-j k_{z} e_{x}-\frac{\partial e_{z}}{\partial x} & =-j \omega \mu_{0} h_{y} \\
j k_{z} h_{y} & =j \omega\left(\varepsilon_{x x} e_{x}+\varepsilon_{x z} e_{z}\right) \\
\frac{\partial h_{y}}{\partial x} & =j \omega\left(\varepsilon_{x z} e_{x}+\varepsilon_{z z} e_{z}\right)
\end{aligned}
$$

and elimination of $e_{x}$ and $e_{z}$ in the TM problem leads to

$\frac{\partial^{2} h_{y}}{\partial x^{2}}-2 j k_{z} \frac{\varepsilon_{x z}}{\varepsilon_{x x}} \frac{\partial h_{y}}{\partial x}+\left[k_{0}^{2} \frac{\varepsilon_{x x} \varepsilon_{z z}-\varepsilon_{x z}^{2}}{\varepsilon_{x x}}-k_{z}^{2} \frac{\varepsilon_{z z}}{\varepsilon_{x x}}\right] h_{y}=0$.
Substituting the solution $\exp (\alpha x)$ in the TM equation leads to the following solutions for $\alpha$

$$
\alpha_{\mathrm{TM}}=j k_{z} \frac{\varepsilon_{x z}}{\varepsilon_{x x}} \pm \frac{\sqrt{\varepsilon_{x x} \varepsilon_{z z}-\varepsilon_{x z}^{2}}}{\varepsilon_{x x}} \sqrt{k_{z}^{2}-\varepsilon_{x x} k_{0}^{2}} .
$$

This equation shows that the TM mode displays a linear phase change along the thickness of the slab. This is analog to the double refraction that causes a change in propagation direction between the ordinary and extraordinary wave in a uniform uniaxial medium. When we consider a symmetric slab waveguide with an isotropic cladding $\varepsilon_{1}$ and apply the boundary conditions for the transverse field components the following dispersion relation is obtained:

$$
\tan \chi a=\frac{2 \varepsilon_{1} \varepsilon^{\prime} \chi \delta}{\varepsilon_{1}^{2} \chi^{2}-\varepsilon^{\prime 2} \delta^{2}}
$$

In this equation $\chi=\sqrt{\varepsilon_{x x} \varepsilon_{z z}-\varepsilon_{x z}^{2}} \sqrt{\varepsilon_{x x} k_{0}^{2}-k_{z}^{2}} / \varepsilon_{x x}, \delta=$ $\sqrt{k_{z}^{2}-\varepsilon_{1} k_{0}^{2}}$ and $\varepsilon^{\prime}=\left(\varepsilon_{x x} \varepsilon_{z z}-\varepsilon_{x z}^{2}\right) / \varepsilon_{x x}$ and $a$ is the thickness of the core layer. This dispersion relation results in a discrete set of values for $k_{z}$. The guided modes must fulfill the following condition: $\sqrt{\varepsilon_{1}} k_{0}<k_{z}<\sqrt{\varepsilon_{x x}} k_{0}$

\section{REFERENCES}

[1] N. Clark and M. Handshy, "Surface-stabilized ferroelectric liquid-crystal electro-optic waveguide switch," Appl. Phys. Lett., vol. 57, pp. 1852-1854, 1990.

[2] A. d'Alessandro and R. Asquini, "Liquid crystal devices for photonic switching applications: State of the art and future developments," Mol. Cryst. Liq. Cryst., vol. 398, pp. 207-221, 2003. 
[3] H. Desmet, W. Bogaerts, A. Adamski, J. Beeckman, K. Neyts, and R. Baets, "Silicon-on-insulator optical waveguides with liquid crystal cladding for switching and tuning," in Proc. ECOC, 2003, vol. 3, pp. $430-431$.

[4] L. Sirleto, G. Coppola, and G. Breglio, "Optical multimode interference router based on a liquid crystal waveguide," J. Opt. A: Pure Appl. Opt., vol. 5, pp. S298-S304, 2003.

[5] C. Gizzi, R. Asquini, and A. d'Alessandro, "A polarization independent liquid crystal assisted vertical coupler switch," Mol. Cryst. Liq. Cryst., vol. 421, pp. 95-105, 2004.

[6] A. d'Alessandro, B. Bellini, D. Donisi, R. Beccherelli, and R. Asquini, "Nematic liquid crystal optical channel waveguides on silicon," IEEE J. Quantum Electron., vol. 42, no. 9-10, pp. 1084-1090, Sep. 2006.

[7] A. Fratalocchi, R. Asquini, and G. Assanto, "Integrated electro-optic switch in liquid crystals," Opt. Exp., vol. 13, pp. 32-36, 2005.

[8] S. V. Serak, N. V. Tabiryan, M. Peccianti, and G. Assanto, "Spatial soliton all-optical logic gates," IEEE Photon. Technol. Lett., vol. 18, no. 9-12, pp. 1287-1289, May 2006.

[9] J. Beeckman, K. Neyts, and M. Haelterman, "Patterned electrode steering of nematicons," J. Opt. A: Pure Appl. Opt., vol. 8, pp. 214-220, 2006.

[10] J. Henninot, M. Debailleul, R. Asquini, A. d'Alessandro, and M. Warenghem, "Self-waveguiding in an isotropic channel induced in dye doped nematic liquid crystal and a bent self-waveguide," J. Opt. A: Pure Appl. Opt., vol. 6, pp. 315-323, 2004.

[11] R. James, E. Willman, F. A. Fernández, and S. E. Day, "Finite-element modeling of liquid-crystal hydrodynamics with a variable degree of order," IEEE Trans. Electron Dev., vol. 53, no. 7, pp. 1575-1582, Jul. 2006.

[12] P. G. de Gennes and J. Prost, The Physics of Liquid Crystals, ser. International Series of Monographs on Physics. Oxford, U.K.: Oxford Univ. Press, 1995.

[13] R. Asquini and A. d'Alessandro, "Bpm analysis of an integrated optical switch using polymeric optical waveguides and ssflc at $1.55 \mu \mathrm{m}, " \mathrm{Mol}$. Cryst. Liq. Cryst, vol. 375, pp. 243-247, 2002.

[14] G. Ntogari, D. Tsipouridou, and E. Kriezis, "A numerical study of optical switches and modulators based on ferroelectric liquid crystals," $J$. Opt. A: Pure Appl. Opt., vol. 7, pp. 82-87, 2005.

[15] E. Gros and L. Dupont, "Beam deflector using double-refraction in ferroelectric liquid crystal waveguides," Ferroelectrics, vol. 246, pp. 219-226, 2000.

[16] J. Beeckman, K. Neyts, X. Hutsebaut, C. Cambournac, and M. Haelterman, "Simulation of 2-D lateral light propagation in nematic-liquidcrystal cells with tilted molecules and nonlinear reorientational effect," Opt. Quant. Electron., vol. 35, pp. 95-106, 2005.

[17] J. Beeckman, K. Neyts, X. Hutsebaut, and M. Haelterman, "Observation of out-coupling of a nematicon," Opto-Electron. Rev., vol. 14, no. 4, pp. 263-267, 2006, 12.

[18] S. Selleri, L. Vincetti, A. Cucinotta, and M. Zoboli, "Complex FEM modal solver of optical waveguides with PML boundary conditions," Opt. Quant. Electron., vol. 33, pp. 359-371, 2001.

[19] A. Fallahkhair, S. Li, and T. Murphy, "Vector finite difference modesolver for anisotropic dielectric waveguides," J. Lightw. Technol., vol. 26, no. 11, pp. 1423-1431, Jun.1 2008.

[20] L. Valor and J. Zapata, "An efficient finite element formulation to analyze waveguides with lossy inhomogeneous bi-anisotropic materials," IEEE Trans. Microw. Theory Tech., vol. 44, no. 2, pp. 291-296, Feb. 1996.

[21] S. Hsu, M. Chen, and H. Chang, "Investigation of band structures for 2D non-diagonal anisotropic photonic crystals using a finite element method based eigenvalue algorithm," Opt. Exp., vol. 15, pp. 5416-5430, 2007.

[22] V. Schulz, "Adjoint high-order vectorial finite elements for nonsymmetric transversally anisotropic waveguides," IEEE Trans. Microw. Theory Tech., vol. 51, no. 4, pp. 1086-1095, Apr. 2003.

[23] F. Bertazzi, O. Peverini, M. Goano, G. Ghione, R. Orta, and R. Tascone, "A fast reduced-order model for the full-wave fem analysis of lossy inhomogeneous anisotropic waveguides," IEEE Trans. Microw. Theory Tech., vol. 50, no. 9, pp. 2108-2114, Sep. 2002.

[24] P. Savi, I. Gheorma, and R. Graglia, "Full-wave high-order FEM model for lossy anisotropic waveguides," IEEE Trans. Microw. Theory Tech., vol. 50, no. 2, pp. 495-500, Feb. 2002.

[25] Y. Lu and F. A. Fernandez, "An efficient finite element solution of inhomogeneous anisotropic and lossy dielectric waveguides," IEEE Trans. Microw. Theory Tech., vol. 41, no. 6, pp. 1215-1223, Jun./Jul. 1993.
[26] J. A. M. Svedin, "Propagation analysis of chirowaveguides using the finite element method," IEEE Trans. Microw. Theory Tech., vol. 38, no. 10 , pp. 1488-1496, Oct. 1990.

[27] J. A. M. Svedin, "A numerically efficient finite-element formulation for the general waveguide problem without spurious modes," IEEE Trans. Microw. Theory Tech., vol. 37, no. 11, pp. 1708-1715, Nov. 1989.

[28] T. Angkaew, M. Matsuhara, and N. Kumagai, "Finite-element analysis of waveguide modes: A novel approach that eliminates spurious modes," IEEE Trans. Microw. Theory Tech., vol. MTT-35, no. 2, pp. 117-123, Feb. 1987.

[29] L. Valor and J. Zapata, "Efficient finite element analysis of waveguides with lossy inhomogeneous anisotropic materials characterized by arbitrary permittivity and permeability tensors," IEEE Trans. Microw. Theory Tech., vol. 43, no. 10, pp. 2452-2459, Oct. 1995.

[30] G. Tartarini and H. Renner, "Efficient finite-element analysis of tilted open anisotropic optical channel waveguides," IEEE Trans. Microw. Theory Tech., vol. 9, no. 10, pp. 389-391, Oct. 1999.

[31] L. Nuno, J. Balbastre, and H. Castane, "Analysis of general lossy inhomogeneous and anisotropic waveguides by the finite-element method (FEM) using edge elements," IEEE Trans. Microw. Theory Tech., vol. 45, no. 3, pp. 446-449, Mar. 1997.

[32] S. Hsu and H. Chang, "Full-vectorial finite element method based eigenvalue algorithm for the analysis of 2D photonic crystals with arbitrary 3d anisotropy," Opt. Exp., vol. 15, pp. 15 797-15 811, 2007.

[33] J. Jin, The Finite Element Method in Electromagnetics, 2nd ed. New York: Wiley, 2002.

[34] M. Koshiba and Y. Tsuji, "Curvilinear hybrid edge/nodal elements with triangular shape for guided-wave problems," J. Lightw. Technol., vol. 18, no. 5, pp. 737-743, May 2000.

[35] F. Tisseur and K. Meerbergen, "The quadratic eigenvalue problem," SIAM Rev., vol. 43, pp. 235-286, 2001.

[36] A. F. Peterson, "Vector finite element formulation for scattering from two-dimensional heterogeneous bodies," IEEE Trans. Antenna Propag., vol. 43, no. 3, pp. 357-365, Mar. 1994.

[37] V. Chigrinov, V. Kozenkov, and H. Kwok, Photoalignment of Liquid Crystalline Materials, ser. Wiley Series in Display Technology. New York: Wiley, 2008.

[38] H. Desmet, K. Neyts, and R. Baets, "Modeling nematic liquid crystals in the neighborhood of edges," J. Appl. Phys., vol. 98, p. 123517, 2005.

[39] P. Yeh and C. Gu, Optics of Liquid Crystal Displays. New York: Wiley-Interscience, 1999.

[40] Y. Satomura, M. Matsuhara, and N. Kumagai, "Analysis of electromagnetic-wave modes in anisotropic slab waveguide," IEEE Trans. Microw. Theory Tech., vol. MTT-22, no. 2, pp. 86-92, Feb. 1974.

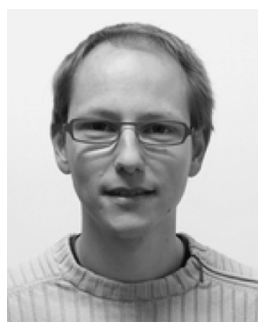

Jeroen Beeckman was born in Zottegem, Belgium, in 1979. He received the M.S. degree in physics engineering in 2002 and the Ph.D. degree in 2006 from the Faculty of Engineering, Ghent University, Gent, Belgium.

Currently, he is a Postdoctoral Fellow of the Research Foundation-Flanders. He is working in the Liquid Crystals and Photonics Group, Department of Electronics and Information Systems. During his postdoctoral research period, he worked 6 months in the Service d'Optique et Acoustique, Université Libre de Bruxelles, where he did research on optical modulation instability. $\mathrm{He}$ also worked 6 months at the Department of Electronics and Electrical Engineering, University College London, on modelling of liquid crystal waveguides. His main research topic is optics of liquid crystals and in particular the development of reconfigurable optical interconnections with the use of liquid crystals.

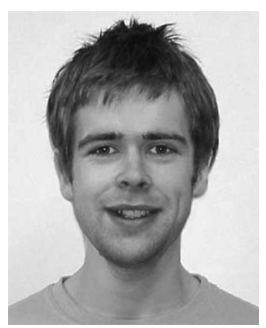

Richard James received the M.Eng. degree in electronic and electrical engineering and the Ph.D. degree from the University College London, London, U.K., in 2001 and 2006, respectively.

$\mathrm{He}$ is working as a Research Fellow in the Liquid Crystal Modelling Group, University College London. His research interests include modeling of LC devices for displays and communications. 


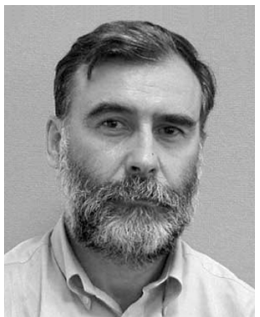

F. Aníbal Fernández (M’88) received the B.Sc. degree in applied mathematics from Universidad de Chile, Santiago, Chile, in 1969 and the Ph.D. degree in electrical engineering from the University College London, London, U.K., in 1981.

Until 1985, he was on the Academic Staff of the Department of Electrical Engineering, Universidad de Chile. In 1986, he joined the staff of the Department of Electronic and Electrical Engineering, University College London, where he is currently a Senior Lecturer. His research interests include microwave and optical aspects of electromagnetic theory, especially the use of numerical methods in those fields. In the last ten years, he has concentrated mainly on the modeling of LC devices.

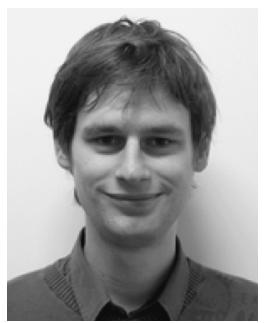

Wout De Cort was born in Asse, Belgium, in 1983. He received the M.S. degree in science of engineering: photonics from Ghent University, Gent, Belgium, in July 2006.

Since then he has been working as a Researcher/Ph.D. student at Ghent University. His work is done partly in the Liquid Crystals and Photonics Group and partly in the Photonics Research Group. This has lead to a modest number of publications in international conferences. His main focus of investigation is the integration of liquid crystals and luminescent nanoparticles with integrated optics, more in particular silicon photonics.

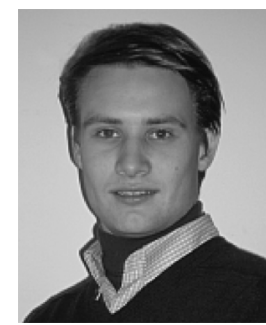

Pieter J. M. Vanbrabant was born in Waregem, Belgium, in August 1984. He received the degree in electrical engineering (summa cum laude) from Ghent University, Gent, Belgium, in July 2007. Since October 2007, he has been working towards the $\mathrm{Ph} . \mathrm{D}$. degree in electrical engineering in the Liquid Crystals and Photonics Group, Ghent University.

His current research interests include the optical modeling of diffractive optics applications and ultrawide viewing LCDs.

Mr. Vanbrabant was awarded by the Research Foundation-Flanders (FWO Vlaanderen) with the Barco student prize.

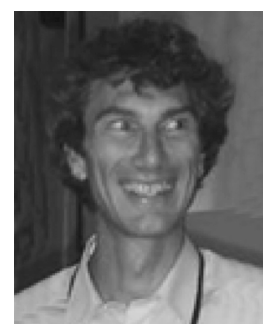

Kristiaan Neyts was born in Oostende, Belgium, in 1964. He received the B.S. degree in physics engineering in 1987 and the Ph.D. degree in engineering in 1992, both from the Faculty of Engineering, Ghent University, Gent, Belgium.

$\mathrm{He}$ was with the Flemish Fund for Scientific Research from 1988 to 2000, when he became a full-time Professor in the Electronics and Information Systems Department (ELIS), Ghent University. $\mathrm{He}$ has been involved in the research of ac high-field electroluminescent displays, light emission from microcavity structures, and electro-optic behavior of display devices. He was on leave in 1987-1988 at the Institute for Physical Electronics, Stuttgart, Stuttgart, Germany, and in 1996-1997 at the Lawrence Berkeley National Laboratory, Berkeley, CA. Since 2000, he has been heading the Liquid Crystals and Photonics Group, ELIS, Ghent University. 